\title{
THE IMPACT OF HOMOPHILY AND HERDING ON DECISION CONFIDENCE IN SOCIAL COMMERCE
}

\author{
Mariam Munawar, Khaled Hassanein and Milena Head \\ DeGroote School of Business \\ McMaster University \\ 1280 Main St. West, Hamilton, ON, Canada
}

\begin{abstract}
Social commerce is a new variant of e-commerce, fitted with social media technologies that allow users to interact with each other. These interactions may facilitate feelings of homophily, and may propagate herd behaviour within the online medium. This work-in-progress paper hones in on how homophily and herding arise within the social commerce context, and examines how it impacts a consumer's' decision-making process. A research model is proposed and an experimental methodology is outlined. Potential contributions to both theory and practice are discussed.
\end{abstract}

\section{KEYWORDS}

Homophily, Herd Behaviour, E-Commerce, Social Commerce

\section{INTRODUCTION}

Online shopping is experiencing exponential growth, with forecasts suggesting almost a $276.9 \%$ increase in the next couple years, culminating in a $\$ 4.9$ trillion-dollar industry by 2021 (Orendorff 2019). This rapid uptake can be attributed to the emergence of social commerce (s-commerce) (Ramachandran 2018), a new variant of e-commerce fitted with social media tools that facilitate social interaction and sharing (Zhang and Benyoucef 2016). However, despite the growing popularity of this medium, shopping online is fraught with uncertainty, given the spatial and temporal separation between consumer and vendor (Pavlou et al. 2007). This is further complicated by the bells and whistles associated with the online space, resulting in information overload and complexity (Rodrigues 2018). These issues with online shopping can have a dire impact on a consumer's decision-making process (Gao et al. 2012), shaking his/her confidence in a purchase decision (Lee and Lee 2004). Although research in social commerce is gaining momentum (see Zhang and Benyoucef 2016), particularly in regards to consumers' buying intentions (eg: Hajli et al. 2017), one's confidence in his/her decisions through this medium has yet to be explored.

Decision confidence is a critical construct in understanding consumer behaviour and is important in understanding an individual's decision-making process (Oney and Oksuzoglu-Guven 2015). It is an important factor in influencing attitude formation and purchase intentions (Bennet and Harrel 1975), and has been suggested to reduce purchase uncertainty, anxiety, and post-purchase dissonance (Keng and Liao 2013). It is a predictor of future buyer behaviour (Chakrabarty et al. 1998), a predictor of household consumption (Dees and Brinca 2013), and an antecedent of brand commitment (Sung \& Choi 2010). As can be seen through the literature, decision confidence is a critical construct that impacts an individual's behaviour, and investigating it within the social commerce context is both timely and needed.

Decision confidence is the belief about the goodness of one's judgments or choices, and has been described as the converse of subjective uncertainty in one's decisions (Sniezek 1992). One approach to reducing subjective uncertainty online, and thereby increasing confidence in one's decision, may be through identification with a group of similar others. This proposition is extrapolated from uncertainty-identity theory (Hogg 2000) which posits that individuals are able to overcome feelings of uncertainty by self-identifying with a group with whom they share similar attributes. Homophily is the extent to which individuals perceive themselves to be similar to others in either interests or demographics (McPherson et al. 2001). Research indicates that, in addition to reducing uncertainty, homophily also increases trust in others' recommendations 
or suggestions (Golbeck 2009). If individuals are able to perceive homophily within the online shopping space, it may be a valuable asset in aiding the decision-making process and building decision confidence.

The unique design of the s-commerce interface may facilitate perceptions of homophily. The s-commerce platform is integrated with specialized tools known as social media markers (SMM) which measure and track the activities and attributes of online consumers, providing convenient statistics on various measures, such as the total number of purchases or recommendations for a product (Munawar et al. 2017). Advances in data-mining technologies are making the design of SMMs more personalized, and these are now capable of providing information on the degree to which consumers are similar to one another in terms of interests and demographics (see Zafarani et al. 2014). There has been limited empirical research on the use of homophily within the social commerce context, and SMMs indicating measures of similarity are only just emerging.

SMM's are useful tools because they serve as heuristics, simplifying the complexity of online information overload. They are also partly responsible for the emergence of a phenomenon known as herd behaviour (Munawar et al. 2017), wherein individuals make decisions based on the observation of others' actions (Bikhchandani et al. 1992). Herd behaviour is quite prevalent in online shopping, due to the widespread use of SMMs that allow individuals to observe consumers' aggregated purchase-related information (such as the total number of recommendations or likes for a product). Its pervasiveness has led to a steady rise in empirical research on its occurrence in social commerce (Wang and Yu 2017); however, the research is sparse, and there remains a critical gap in understanding the interaction of herd behaviour with homophily, and its combined impact on the decision-making process.

Given the context outlined above, the purpose of this study is to build a more comprehensive understanding of a consumer's decision-making process by mapping the factors impacting decision confidence within the realm of social commerce. An attempt is made to uncover the elements of the s-commerce interface that serve as mechanisms for uncertainty reduction. In particular, this study focuses on the impact of homophily in reducing uncertainty, and in its interaction with herd behaviour to drive one's decision confidence.

\section{RESEARCH MODEL}

Our research model is rooted in the postulates of uncertainty-identity theory (UIT) (Hogg 2000; 2007) which suggests that uncertainty is an aversive state and that people are driven to reduce it. Identification with a group of others can serve as a means for uncertainty reduction because it allows for the development of a social comparative context that validates one's self concept, behaviours, and cognitions. In using UIT as a framework for our model, we are able to tease out perceived homophily (captured as interests homophily and demographic homophily) and herd size as factors impacting decision confidence as both these constructs aid in reducing subjective uncertainty within the online shopping context. UIT provides a theoretical underpinning for our research model, allowing us to integrate it with trust and sense of community as mediators that facilitate the reduction of uncertainty and the development of decision confidence. These mediators have been identified through their relationship to uncertainty reduction, as well as their relationship to homophily and herding.

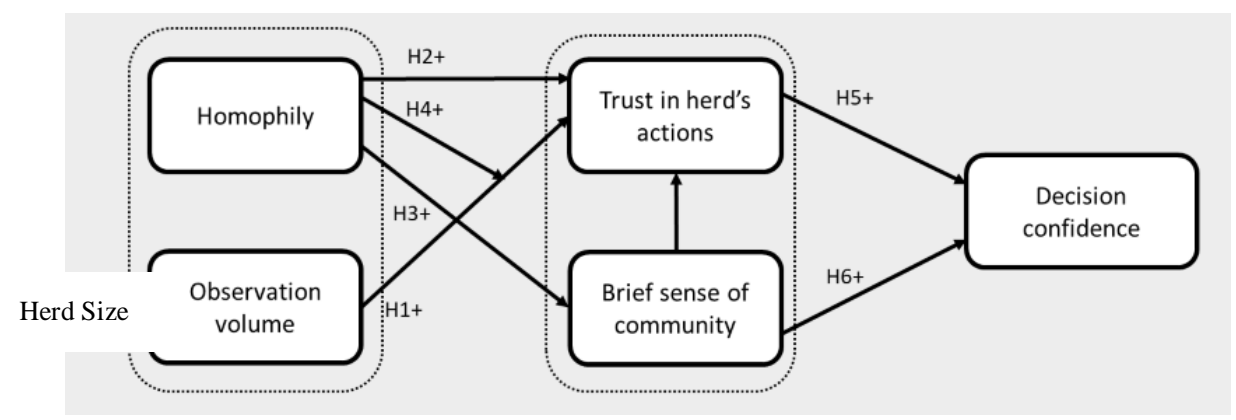

Figure 1. Research Model 


\section{RESEARCH METHOLOGY}

The proposed program will be validated using an experimental study followed by administration of a survey. A factorial design will be employed to assess the main and interaction effects of homophily and herding on an individual's decision confidence within the social commerce context. Herding is captured through herd size, and will be operationalized as the total number of recommendations made for a product/service. Research indicates that herd size is an important factor in driving herd behaviour, and various studies report that the number of individuals in a herd will impact the likelihood of others joining and conforming to the actions of the collective. Homophily will be operationalized using a homophily index that will be presented as a percentage indicating similarity to consumers who are making these recommendations. Homophily is operationalized with four incremental levels (including a control zero level), based on the type of homophily being employed: (1) no homophily index; (2) demographic homophily; (3) interests-based homophily; or (4) both demographic and interest-based homophily. Interests-based homophily has been shown to be more potent in influencing behaviour than demographics-based homophily. Research also indicates that the combined impact of both may have stronger effects than either alone (Ensher et al. 2002).

Table 1. Research Design

\begin{tabular}{|l|l|l|l|l|}
\hline \multirow{2}{*}{ Herd Size } & \multicolumn{4}{|c|}{ Homophily } \\
\cline { 2 - 5 } & No homophily & $\begin{array}{l}\text { Homophily } \\
\text { Demographics }\end{array}$ & Homophily Interests & Homophily D + I \\
\hline Small & 40 & 40 & 40 & 40 \\
\hline Large & 40 & 40 & 40 & 40 \\
\hline
\end{tabular}

A fictitious website will be created where various treatments of these SSMs will be manipulated. Participants will view the homepage of the site, which will vary in these markers according to the experimental design. The website will be developed to simulate a social commerce platform, and subjects will be provided with a specific shopping related task. The research design will follow the approach utilized in other experimental studies on information seeking and decision-making behaviour (Tan, Teo and Benbasat, 2010), and will allow for the induction of mundane realism (Tan et al. 2010) to provide a realistic scenario. 40 participants will be randomly assigned to each treatment, for a total sample size of 320 subjects. After viewing the experimental site and completing the task, subjects will be administered a survey to answer questions about the various constructs utilized in the study.

\section{PROPOSED ANALYSES}

The research model will be analyzed using structural equation modeling (SEM) because of its ability to simultaneously assess both the measurement and structural model components, thereby resulting in a more rigorous methodological analysis (Gefen et al. 2011). The Partial Least Squares method will be applied because of its ability to allow modeling of latent constructs under conditions of non-normality and small-medium sample sizes. In an effort to triangulate results, a qualitative component will be included through use of open-ended questions to allow for more in-depth explanations and clarifications of the participants' experience. Content analysis techniques will be applied to identify emerging trends and patterns.

\section{CONCLUSION}

This research will aid in developing a clearer picture of a consumer's purchase behaviour through this medium. Because of the rising popularity of s-commerce, it is critical for vendors to understand how individuals make decisions in this setting, and the variables impacting their confidence, because of the central role confidence plays in driving satisfaction and channel adoption. In explicating the variables impacting decision confidence in social commerce, this research aids in bridging a gap in the academic literature; it also provides vendors with practical and relevant suggestions for improving the overall buying experience of its consumers. 


\section{REFERENCES}

Bennett, P. D., \& Harrell, G. D. (1975). The role of confidence in understanding and predicting buyers' attitudes and purchase intentions. Journal of Consumer Research, 2(2), 110-117.

Bikhchandani, S., Hirshleifer, D., \& Welch, I. (1992). A theory of fads, fashion, custom, and cultural change as informational cascades. Journal of political Economy, 992-1026.

Chakrabarty, S., Chopin, M., \& Darrat, A. (1998). Predicting future buyer behavior with consumers' confidence and sentiment indexes. Marketing Letters, 9(4), 349-360.

Dees, S., \& Brinca, P. S. (2013). Consumer confidence as a predictor of consumption spending: Evidence for the United States and the Euro area. International Economics, 134, 1-14.

Gao, J., Zhang, C., Wang, K., \& Ba, S. (2012). Understanding online purchase decision making: The effects of unconscious thought, information quality, and information quantity. Decision Support Systems, 53(4), 772-781.

Gefen, D., Rigdon, E. E., \& Straub, D. (2011). Editor's comments: an update and extension to SEM guidelines for administrative and social science research. Mis Quarterly, iii-xiv.

Golbeck, J. (2009). Trust and nuanced profile similarity in online social networks. ACM Transactions on the Web (TWEB), 3(4), 12.

Hajli, N., Sims, J., Zadeh, A. H., \& Richard, M. O. (2017). A social commerce investigation of the role of trust in a social networking site on purchase intentions. Journal of Business Research, 71, 133-141.

Hogg, M. A. (2000). Subjective uncertainty reduction through self-categorization: A motivational theory of social identity processes. European review of social psychology, 11(1), 223-255.

Hogg, M. A. (2007). Uncertainty-identity theory. Advances in experimental social psychology, 39, 69-126.

Keng, C. J., \& Liao, T. H. (2013). Self-confidence, anxiety, and post-purchase dissonance: a panel study. Journal of Applied Social Psychology, 43(8), 1636-1647.

Lee, B. K., \& Lee, W. N. (2004). The effect of information overload on consumer choice quality in an on-line environment. Psychology \& Marketing, 21(3), 159-183.

McPherson, M., Smith-Lovin, L., \& Cook, J. M. (2001). Birds of a feather: Homophily in social networks. Annual review of sociology, 27(1), 415-444.

Munawar, M., Hassanein, K., \& Head, M. (2017). Understanding the Role of Herd Behaviour and Homophily in Social Commerce. SIGHCI 2017 Proceedings. 11.

Oney, E., \& Oksuzoglu-Guven, G. (2015). Confidence: A critical review of the literature and an alternative perspective for general and specific self-confidence. Psychological reports, 116(1), 149-163.

Orendorff, A. (2017). Global Ecommerce: Statistics and International Growth Trends. Shopify-Plus. Retrieved from: https://www.shopify.com/enterprise/global-ecommerce-statistics

Pavlou, P. A., Liang, H., \& Xue, Y. (2007). Understanding and mitigating uncertainty in online exchange relationships: A principal-agent perspective. MIS quarterly, 105-136.

Ramachandran, M. (2018). The Evolution of Social Shopping in the Ecommerce Landscape. Retrieved from: https://www.adweek.com/digital/the-evolution-of-social-shopping-in-the-ecommerce-landscape/

Rodrigues, A. (2018). Our Brains Are Being Overloaded With Push Notifications About Nothing. Motherboard: Vice. Retrieved from: https://motherboard.vice.com/en_us/article/a3a848/facebook-notification-overload

Sniezek, J. A. (1992). Groups under uncertainty: An examination of confidence in group decision making. Organizational Behavior and Human Decision Processes, 52(1), 124-155.

Sung, Y., \& Choi, S. M. (2010). "I won't leave you although you disappoint me": The interplay between satisfaction, investment, and alternatives in determining consumer-brand relationship commitment. Psychology \& Marketing, 27(11), 1050-1073.

Tan, C. H., Teo, H. H., \& Benbasat, I. (2010). Assessing screening and evaluation decision support systems: A resource-matching approach. Information Systems Research, 21(2), 305-326.

Wang, Q., \& Shukla, P. (2013). Linking sources of consumer confusion to decision satisfaction: the role of choice goals. Psychology \& Marketing, 30(4), 295-304.

Wang, Y., \& Yu, C. (2017). Social interaction-based consumer decision-making model in social commerce: The role of word of mouth and observational learning. International Journal of Information Management, 37(3), 179-189.

Zafarani, R., Abbasi, M. A., \& Liu, H. (2014). Social media mining: an introduction. Cambridge University Press.

Zhang, K. Z., \& Benyoucef, M. (2016). Consumer behavior in social commerce: A literature review. Decision Support Systems, 86, 95-108. 